\title{
Primary Intra-Osseous Basaloid Squamous Cell Carcinoma of Mandible: Report of Rare Case with Proposed Diagnostic Criteria
}

\author{
Manas Bajpai, Betina Chandolia, Nilesh Pardhe and Manika Arora \\ Department of Oral Pathology, NIMS Dental College, Jaipur, India
}

\begin{abstract}
Basaloid squamous cell carcinoma (BSCC) is a distinctive variant of squamous cell carcinoma (SCC) characterised by nests of basaloid cells. BSCC is an aggressive and rare tumor of head and neck region; and is relatively rare in oral cavity in comparison to SCC. Although, infrequent, but primary intra-osseous SCC (PIOSCC) has been reported in the jaws. There are separate diagnostic criteria for this lesion and a classification for primary intra-osseous carcinoma has been described. No evidence was found in the published literature about primary intra-osseous BSCC (PIOBSCC).

We, herein, present a case of giant osteodestructive lesion of the posterior mandible which was diagnosed as PIOBSCC with a correlation of histopathological and immunohistochemical features. As per the best of our knowledge, this is the first case of PIOBSCC of the jaw. There is no diagnostic criterion of PIOBSCC in the literature owing to the extreme rarity of this tumor. A diagnostic criterion is proposed here to make the diagnosis of this tumor easier.
\end{abstract}

Key Words: Basaloid squamous cell carcinoma, Intra-osseous, Histopathology, Mandible.

How to cite this article: Bajpai M, Chandolia B, Pardhe N, Arora M. Primary intra-osseous basaloid squamous cell carcinoma of mandible: Report of rare case with proposed diagnostic criteria. J Coll Physicians Surg Pak 2019; 29(12):1215-1217.

\section{INTRODUCTION}

Basaloid squamous cell carcinoma (BSCC), defined by $\mathrm{WHO}$ as an aggressive high grade variant of squamous cell carcinoma (SCC), is infrequent in oral cavity and relatively more common in the oropharynx. ${ }^{1}$ Histopathologically, the tumor is comprised of both basaloid and squamous components. BSCC is commonly found in head and neck region and the common sites include epiglottis, and pyriform sinuses. ${ }^{2}$ Intra-orally, cases have been reported in the base of the tongue, floor of the mouth, palate, buccal mucosa, and gingiva. ${ }^{3}$ An exhaustive literature review revealed no case of BSCC in the jaw bone. To the best of our knowledge, the present case is the first repeated example of primary intra-osseous BSCC (PIOBSCC).

\section{CASE REPORT}

An otherwise healthy 22-year male presented to our outpatient department with the chief complaint of painful swelling in left back region of the jaw for 8 months. The swelling was initially small and painless that gradually increased in size. Family history and past medical history of the patient were not relevant to the presenting symptoms. Personal history of the patient revealed that he was a chronic smoker and consumed $10-15$ cigarettes per day for 4 years. Extra-oral examination of

Correspondence to: Dr. Betina Chandolia, NIMS Dental College, NIMS University, Jaipur, India

E-mail:drbetina24@gmail.com

Received: January 23, 2019; Revised: March 14, 2019;

Accepted: March 30, 2019 the patient revealed an asymmetrical facial profile with large swelling on the left side of the face (Figure 1). The swelling was firm on palpation. The overlying skin on the swelling was similar to the adjacent skin without any sign of discharge and ulceration.

Left submandibular lymph nodes were enlarged, palpable, mobile and tender. Intra-oral examination revealed a bony hard swelling extending from the gingiva of tooth \#33 to the ramus of the mandible measuring about $3 \times 2 \mathrm{~cm}$. No ulceration and/or discharging sinus was found. Panoramic radiograph revealed a large multilocular radiolucent lesion extending from the body of the mandible to the neck of the condyle at one end and coronoid process at the other end. Partially, erupted tooth \#36 was found to be engulfed inside the radiolucency (Figure 2). An incisional biopsy of the lesion was performed and the soft tissue section was sent to the Department of Oral and Maxillofacial Pathology for microscopic evaluation. Microscopic examination of hematoxylin and eosin (H \& E) stained soft tissue sections revealed a connective tissue stroma infiltrated with solid nests of dysplastic basaloid epithelium exhibiting peripheral palisading, cellular and nuclear atypia including cellular pleomorphism, increased nucleo-cytoplasmic ratio and nuclear hyperchromatism. Few abnormal mitotic figures were seen. Keratin pearls were found at places. The solid nests of basaloid epithelium were separated by fibrocellular connective tissue stroma (Figures 3 and 4). The overlying epithelium was severely dysplastic stratified squamous epithelium. No evidence of ameloblastomatous and/or odontogenic cystic epithelium component was found. Immunohistochemical (IHC) 


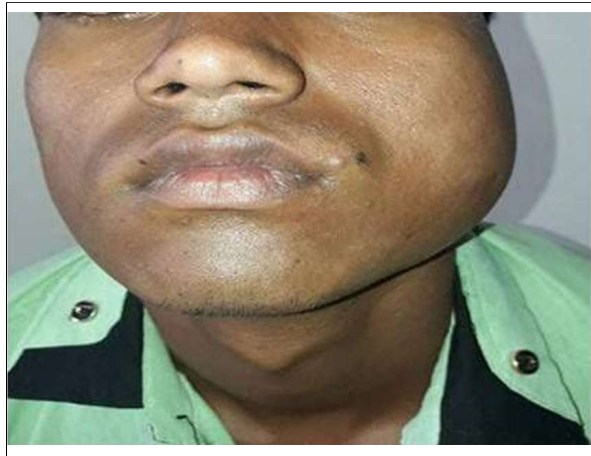

Figure 1: Facial profile of the patient showing a swelling on the left side of the face.

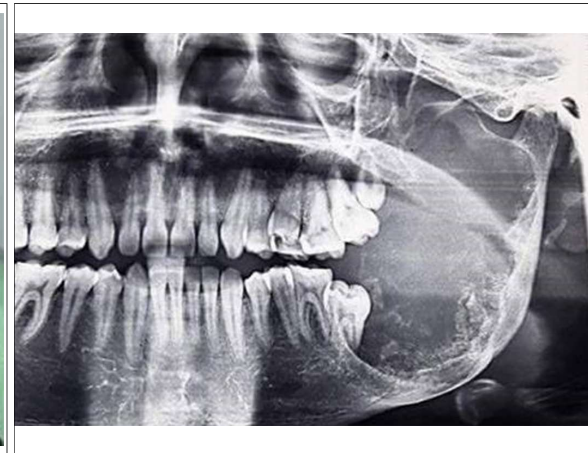

Figure 2: Panoramic radiograph showing a large osteodestructive lesion of the left posterior mandible extending to the coronoid process.

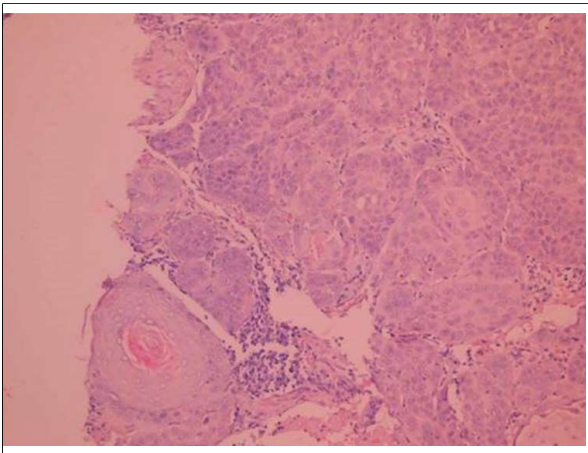

Figure 3: Lobules of basaloid cells separated by fibrocellular stroma (Hematoxylin and Eosin Staining $\times 20$ ).

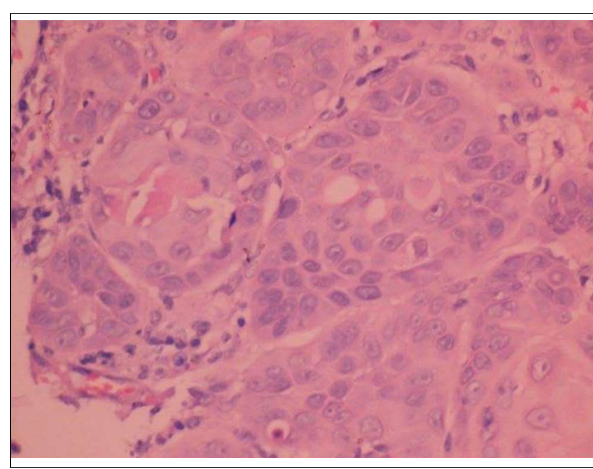

Figure 4: Lobules of dysplastic basaloid cells with some lobules showing cystic degeneration (Hematoxylin and Eosin Staining x 20).

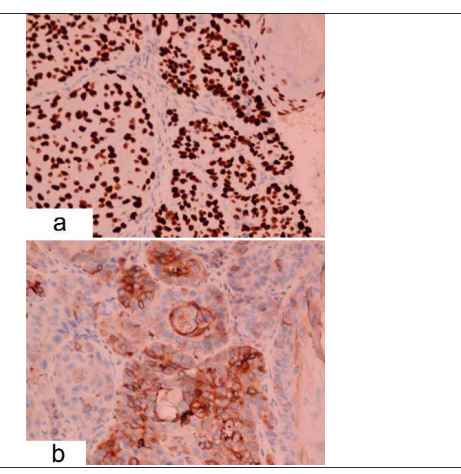

Figure 5: (a) Tumor cells showing diffuse positive expression for P63 (Original magnification x 20). (b) Tumor cells showing positive expression for EMA (Original magnification $\times 20$ ).

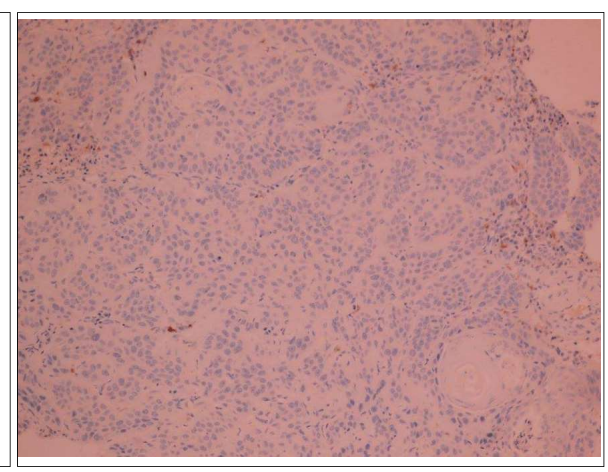

Figure 6: Tumor cells showing negative expression for Ber - EP4 (Original magnification, x 20).

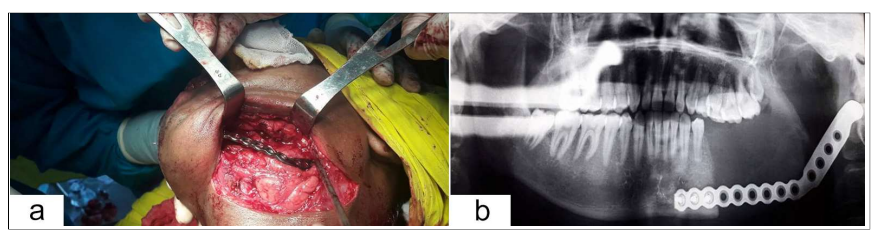

Figure 7: (a) Intra-operative view showing placement of reconstruction plates. (b) Postoperative radiograph after removal of the tumor and reconstruction plate insertion.

examination revealed a strongly positive expression of basaloid cells for $P 63$ (Figure 5a) and epithelial membrane antigen (EMA) (Figure 5b) and negative expression of Ber - EP4 (Figure 6). Based on all these features, a final diagnosis of PIOBSCC was given. Patient was referred to the surgical oncology unit, where left hemimandibulectomy was performed under general anesthesia. After temporary tracheostomy was done, the skin flap was elevated and retracted laterally and superiorly unveiling the mandible (Figure 7a). Left mandible with tumor mass was excised en block with the tumor. The defect was restored by titanium reconstruction plates (Figure $7 \mathrm{~b}$ ). The follow-up period of 2 years was uneventful.

\section{DISCUSSION}

PIOBSCC is a rare tumor. Although cases of BSCC of oral cavity have been reported in the literature in asso- ciation with tongue, gingiva and floor of the mouth 4 , but an exhaustive literature search did not reveal a single case of PIOBSCC of jaw.

BSCC is described as an aggressive variant of SCC associated with relatively poor prognosis. The diagnostic criteria of BSCC are based on histopathological features comprising of focal squamous differentiation, a predominant basaloid pattern that is associated with frank invasive SCC or carcinoma in situ, peripheral palisading, high mitotic rate and solid growth. 5

On the other hand, PIOSCC is described as intra-osseous counterpart of SCC. The diagnostic criteria of PIOSCC of jaws include: absence of ulcer in the overlying mucosa in oral cavity, absence of another primary tumor at the time of diagnosis and for at least 6 months during the follow-up, and histopathological evidence of SCC. Owing to the rarity of PIOBSCC, no diagnostic criteria have been described in the literature. ${ }^{6}$ The diagnostic criteria of PIOBSCC are proposed here (Table I).

The present case fulfilled the diagnostic criteria of BSCC. With the occurrence of the tumor inside the mandible and absence of ulceration in the overlying mucosa and no evidence of other primary tumor, the diagnosis of PIOBSCC was confirmed. The differential diagnoses of PIOBSCC include central adenoid cystic carcinoma $(\mathrm{ACC})$ basal cell ameloblastoma (BCA), central basal 
Table I: A proposed diagnostic criteria for PIOBSCC based on clinical and histopathological features.

1. Absence of ulceration over the underlying mucosa.

2. Radiological evidence of intra-osseous tumor.

3 Absence of another primary tumor at the time of diagnosis and for at least 6 months during the follow-up.

4. Histopathological diagnosis of BSCC including nests of basaloid epithelium with high nuclear cytoplasmic ratio, peripheral palisading, increased mitotic figure, solid growth, associated with SCC without evidence of ameloblastomatous and/or odontogenic cystic epithelium.

cell adenocarcinoma (BCAC), and central basal cell carcinoma (BCC).

PIOBSCC can be differentiated from central ACC by the absence of myoepithellial cells and the presence of laminin and type IV collagen positive material inside cyst-like spaces. Also, ACC does not show dysplasia. Focal necrosis and squamous differentiation usually seen in BSCC, are not seen in BCAC. ${ }^{7}$

This case reports the first case of PIOBSCC of mandible with proposed diagnostic criteria of this rare tumor. We hope these diagnostic criteria will enable the pathologists to diagnose this rare tumor and to differentiate it from its close mimickers.

\section{PATIENT'S CONSENT:}

Consent has been taken from the patient.

\section{CONFLICT OF INTEREST:}

Authors declared no conflict of interest.

\section{AUTHORS' CONTRIBUTION:}

MB, BC, MA: Substantial contributions to the conception or design of the work; or the acquisition, analysis, or interpretation of data for the work, drafting the work or revising it critically for important intellectual content. NP: Final approval of the version to be published.

\section{REFERENCES}

1. Radhi J. Basaloid squamous cell carcinoma. In: Li X, Ed. Squamous Cell Carcinoma. $1^{\text {st }}$ ed. Coratia: Intech publications; 2012.

2. Patil AV, Tupsakhare SD, Shah K, Gabhane M, Ali FM. Basaloid squamous cell carcinoma of gingiva: A new case and review of literature. Am J Cancer Case Rep 2014; 2:99-107.

3. Nemade SV, Rokade VV. Basaloid carcinoma: An unusual presentation. Indian J Clin Pract 2013; 24:33 -7.

4. Wain SL, Kier R, Vollmer RT. Basaloid-squamous carcinoma of the tongue, hypopharynx, and larynx: Report of 10 cases. Hum Pathol 1986; 17:1158-66.

5. Shinno $\mathrm{Y}$, Nagatsuka $\mathrm{H}$, Siar $\mathrm{CH}$. Basaloid squamous cell carcinoma of the tongue in a Japanese male patient: A case report. Oral Oncol Extra 2005; 41:65-9.

6. Suei Y, Tanimoto K, Taguchi A, Wada T. Primary intraosseous carcinoma: Review of the literature and diagnostic criteria. J Oral Maxillofac Surg 1994; 52:580-3.

7. Gopinath D, Beena VT, Stephen M, Sivakumar R, Choudhary K. Basaloid squamous cell carcinoma of oral cavity: Report of two cases. Int J Dent Clin 2012; 4:62-4. 